\title{
Phytodiversité des légumes-feuilles locales consommées par les peuples Holli et Nagot de la région de Pobè au sud-est du Bénin
}

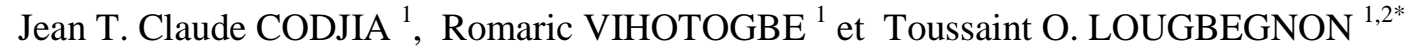 \\ ${ }^{1}$ Département d'Aménagement et Gestion de l'Environnement (D/AGE), Faculté des Sciences Agronomiques \\ (FSA), Université d'Abomey-Calavi (UAC), Bénin. \\ ${ }^{2}$ Laboratoire d'Aménagement des Forêts et de Biogéographie (LAFBio), Cotonou, Bénin. \\ ${ }^{3}$ Ingénieur Agronome; en Aménagement et Gestion des Ressources Naturelles ; PFNL-Labo/FSA/UAC, \\ Cotonou, Bénin. \\ *Auteur correspondant, E-mail : tlougbe@yahoo.fr, BP 526 Cotonou, Bénin.
}

\section{RESUME}

Dans le cadre de la mise en place d'une banque de données fiables propre aux ressources alimentaires locales, la région de Pobè au sud-est du Bénin a été prospectée par l'équipe de recherche du Laboratoire de Gestion des Produits Forestiers Non ligneux de la Faculté des Sciences Agronomiques (PFNLs-Labo/FSA) de l'Université d'Abomey-Calavi du Bénin. Un état des lieux a été fait sur la diversité des espèces de légumesfeuilles locales consommées par les populations Holli et Nagot de cette région. La méthode utilisée par cette étude conduite dans deux villages, repose sur une enquête de consommation alimentaire suivie des relevés en milieu naturel. Au total, 35 espèces végétales ont été identifiées dans les différents faciès végétaux des deux villages. Les Holli ont un spectre alimentaire et une préférence plus larges que les Nagot.

(c) 2009 International Formulae Group. All rights reserved.

Mots clés : Phytodiversité, légumes-feuilles, peuples Holli et Nagot.

\section{INTRODUCTION}

De nombreuses études ont démontré l'importance des produits forestiers non ligneux (PFNL) pour la subsistance des habitants des forêts (Chamber et Leach, 1987). La contribution de ceux-ci à la sécurité alimentaire devient tout à fait évidente, car nombreuses sont les populations des pays en développement qui les utilisent pour se nourrir.

Ces espèces vont des herbacés aux ligneux, et tous les organes sont exploités, partant des racines jusqu'aux fleurs, en passant par les fruits, les feuilles, les écorces, etc. (Herzog, 1992). Au Bénin, ces ressources qui sont longtemps restées hors de toute préoccupation scientifique, suscitent de plus en plus l'intérêt des chercheurs ces dernières années.
Une étude bibliographique des ressources végétales faite par Codjia et al. (2003) au Bénin a révélé l'existence de près de 200 espèces végétales consommées diversement par les populations locales pour leurs fruits, leurs feuilles, leurs racines, leurs fleurs, leurs graines et leurs tubercules. Parmi elles, on note plus de 167 espèces végétales comestibles. La forêt dense semi caducifoliée de Pobè et ses zones connexes offrent d'après Sokpon et Lejoly (1996), une soixantaine d'espèces consommées essentiellement comme fruits et/ou légumes sauvages.

Le présent travail tiré de la base de données sur les produits forestiers alimentaires au Bénin est une contribution à la connaissance des ressources alimentaires des populations rurales Holli et Nagot de Pobè. Elle a permis de mettre en évidence la 
participation effective des légumes-feuilles à l'alimentation des populations rurales au Bénin.

\section{MATERIEL ET METHODES Milieu d'étude}

L'étude s'est déroulée dans la commune de Pobè, qui s'étend entre les parallèles $6^{\circ} 58^{\prime} 04^{\prime \prime}$ ' et 6 $6^{\circ} 57^{\prime} 20^{\prime \prime}$ ' latitude nord et $20^{\circ} 40^{\prime} 45^{\prime}$ ' et $2^{\circ} 39^{\prime} 46^{\prime \prime}$ ' longitude est, au sud-est du Bénin. La collecte des données s'est effectuée principalement dans l'arrondissement rural d'Issaba, situé au cœur de la commune; et dans l'arrondissement de Pobè, fortement urbanisé situé au sud de la commune (Figure 1).

La région de Pobè jouit d'un climat tropical humide à quatre saisons. C'est une région aux caractéristiques climatiques particulières dans le Dahomey-gap, avec une moyenne pluviométrique annuelle pouvant atteindre $1180 \mathrm{~mm}$. Ceci entretient parfaitement la seule forêt dense semi-décidue dont la superficie est passée de 744 ha en 1922 à 115 de nos jours; soit une réduction de $84,5 \%$ de la surface forestière naturelle (Sokpon, 1995). Cette situation pluviométrique avec les deux saisons de pluies entretient deux cycles culturaux avec deux cycles végétatifs pour les adventices. La végétation est une mosaïque de reliques forestières fortement dégradées, de champs, de jachères et de plantations de toutes sortes.

Deux ethnies (Holli et Nagot) dominent nettement la population. Les activités principales de ces peuples restent l'agriculture itinérante sur brûlis et le commerce des Produits Forestiers Non Ligneux.

\section{Matériel d'étude}

Le matériel d'étude était constitué de : - guides d'identification de la flore Arbonier (2002) pour identifier les espèces;

- de sécateur et des collections d'herbiers de références.

La population d'étude était constituée de deux groupes ethniques majoritaires autochtones et très ruraux de la commune de Pobè que sont les Holli et les Nagot.

\section{Critères de choix des villages ou localités d'étude \\ Les villages d'étude étaient} sélectionnés suivant la prédominance dans la localité de l'un des deux faciès végétaux dominants (jachères et/ou champs et forêt). Ce critère était prépondérant parce que c'est dans ces écosystèmes que les populations vont cueillir les ressources objets d'étude. Ainsi, deux villages représentatifs ont été choisis : le village Issaba (arrondissement d'Issaba essentielle-ment rural) à prédominance Holli $(89 \%)$ entièrement situé dans les jachères au centre de la commune et le village de Talala (dans Pobè-Centre, en milieu rural) à prédominance Nagot $(57 \%)$ et contigu à la forêt au sud de la commune de Pobè.

Cent vingts individus (120) ont été sélectionnés (la sélection de 120 de manière aléatoire dans l'ensemble des deux villages, soit 60 par village). Ces individus ont été répartis dans 20 ménages Nagot ou Holli (10 par village), et étaient composés des personnes des deux sexes appartenant à toutes les catégories.

\section{Méthode d'échantillonnage des données}

Elle était basée sur une enquête de consommation alimentaire auprès des populations suivie d'une prospection de reconnaissance et d'identification systématique des espèces en milieu naturel.

L'enquête était essentiellement basée sur la méthode de rappel de mémoire des individus sur les espèces végétales sauvages consommées comme légumes-feuilles. Au sein de chaque ménage, les individus ont été enquêtés isolément, puis des entretiens de groupe ont été organisés. Un questionnaire structuré a été utilisé.

En outre, après l'enquête auprès des populations, on a procédé à une reconnaissance et identification des espèces en milieu naturel. Une descente s'est effectuée dans les différents faciès végétaux, habitats des diverses espèces de légume-feuille indiquées par les ménages lors des enquêtes de consommation, accompagnée des enfants, des femmes et des personnes ressources (tradithérapeutes) qui maîtrisent parfaitement les lieux de présence de chaque espèce, et les herbiers de référence ont été réalisés à partir des prélèvements d'échantillons. Ces herbiers ont été déposés à l'Herbier National, afin de confirmer les noms scientifiques des espèces et de les joindre aux noms locaux. 


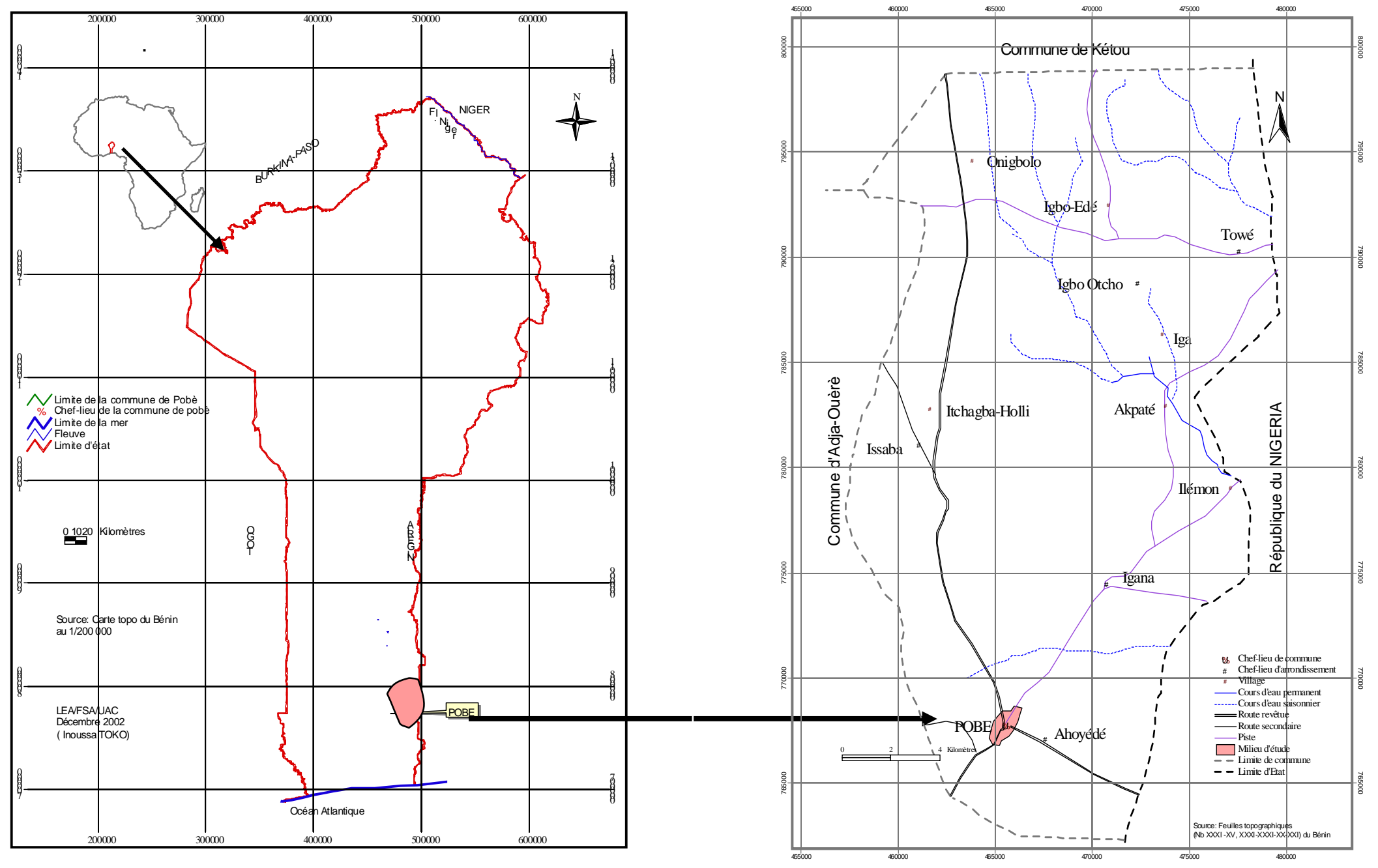

Figure 1: Localisation de la zore d'étude. 
Les données collectées durant ces deux phases d'étude ont concerné surtout les noms locaux en Holi et Nagot et les noms systématiques (famille, genre et espèce) des espèces végétales, leurs types biologique et morphologique, leur disponibilité saisonnière, les fréquences ou degrés de consommation par ethnies, les formes de transformation. Tout ceci a permis de mettre en lumière la diversité des espèces sauvages de légumes-feuilles prélevées dans chaque type de faciès végétal par les ménages, leurs périodes de disponibilité.

\section{Traitement des données collectées}

Dans un premier temps, un aperçu systématique de la phytodiversité des légumes recensés a été dressé avec un classement des espèces selon les noms locaux et systématiques, leurs types biologique et morphologique ainsi que leurs habitats d'occurrence. Ensuite on a calculé pour chacune des espèces, la fréquence $(F i)$ de consommation, qui est le nombre moyen de fois que l'espèce est dite consommée par mois et par individu.

$F_{i}=\sum f_{i} / N$

$f_{i}=$ Fréquence de consommation individuelle $=$ nombre de fois qu'un individu i consomme une espèce donnée par mois,

$N=$ nombre d'individus enquêtés.

\section{RESULTATS}

\section{Diversité des légumes feuilles locales}

$\mathrm{Au}$ total, 35 espèces de légumesfeuilles locales ont été répertoriées dans les deux villages d'étude. Le Tableau 1 en donne les noms vernaculaires, les noms scientifiques, les familles, les types biologiques, les types morphologiques et les habitats. Ces espèces se distribuent dans seize (16) familles dont les Asteraceae (10 espèces), les Amarantaceae (6 espèces) et les Solanaceae (3 espèces) sont les plus contributives $(64 \%$ de la diversité totale). Les 13 autres familles fournissent chacune 1 à 2 espèces. De plus, respectivement $86 \%, 41 \%, 11 \%, 14 \%$, et $8 \%$ des ces espèces proviennent des champs, des jardins de case, des jachères, des plantations et des forêts. Ainsi, plus le milieu est ouvert ou dégradé, plus abondantes sont les espèces de légumes-feuilles locales en son sein.

Quant à la disponibilité saisonnière de ces espèces consommées, le constat est qu'il existe toujours de légumes disponibles quelle que soit la période de l'année. En effet, $42 \%$ des espèces sont disponibles en plein temps pendant toute l'année sans distinction de saison. D'autre part, $58 \%$ des espèces sont présentes de manière continue, sauf pendant une partie de la grande saison sèche. La saison des pluies ne fournit en exclusivité qu'une seule espèce.

\section{Intégrité du savoir endogène et consommation au sein des ethnies}

Toutes les espèces citées par les populations réunies sont présentes dans les différents faciès végétaux des deux villages et sont donc disponibles pour les populations des deux ethnies. La Figure 2 montre le spectre alimentaire en matière de consommation de légumes sauvages des deux ethnies. De l'analyse de cette figure, il ressort que les Holli consomment $100 \%$ des légumes sauvages, alors que les Nagot en consomment les $83 \%$.

Ces espèces sont différemment consommées au sein des populations. Les Figures $3 \mathrm{a}$ et $3 \mathrm{~b}$ présentent l'importance relative des espèces couramment consommées et donc préférées par les ethnies étudiées. L'analyse de ces figures montre une préférence beaucoup plus large des peuples Holli vis-à-vis des légumes locaux. Ainsi, six (6) espèces (Amaranthus spinosu,s, Euphorbia heterophylla, Boerhavia sp, Alternanthera sessilis, Trianthema portulacastrum et Bidens pilosa) sont strictement consommées par les Holli, et figurent encore parmi les espèces préférées.

\section{DISCUSSION}

Nombreuses sont les espèces de légumes locaux répertoriées dans la région de Pobè au sud-est du Bénin. Toutes les formes de vie, partant des herbacées aux grands ligneux sont représentées comme l'a mentionné Herzog (1992). Sokpon et Lejoly (1996) ont observé pour les fruitiers sauvages de la forêt dense semi-décidue de Pobè une diversité de 63 espèces; liste non exhaustive pour toute la région de Pobè. Nos résultats couplés à ceux de Sokpon et Lejoly (1996) montrent une diversité de 98 espèces comestibles pour la seule région de Pobè. Ces 
Tableau 1: Phytodiversité des espèces de légumes-feuilles consommées dans la région de Pobè.

\begin{tabular}{|c|c|c|c|c|c|c|c|}
\hline \multirow{2}{*}{$\begin{array}{l}\text { Numéro } \\
\text { d'ordre }\end{array}$} & \multicolumn{2}{|c|}{ NOMS VERNACULAIRES } & \multirow[t]{2}{*}{ NOMS SCIENTIFIQUES } & \multirow[t]{2}{*}{ FAMILLES } & \multirow{2}{*}{$\begin{array}{l}\text { TYPES } \\
\text { BIOLOGIQUES }\end{array}$} & \multirow{2}{*}{$\begin{array}{l}\text { TYPES } \\
\text { MORPHOLOGIQUES }\end{array}$} & \multirow[t]{2}{*}{ HABITAT } \\
\hline & NAGOT & HOLLI & & & & & \\
\hline 1 & Oché & Oché & Adansonia digitata & Bombacaceae & Phanérophyte & Arbre & Champs \\
\hline 2 & Idé & Idé & Alternantera sessilis & Amarantaceae & Thérophyte & Herbe & Champs \\
\hline 3 & Olokokogbowo & Tètè & Amarantus $s p$ & Amarantaceae & Thérophyte & Herbe & Champs, maisons \\
\hline 5 & Yanrin & Yanrin & Blactuca taraxacifolia & Asteraceae & Thérophyte & Herbe & Champs \\
\hline 6 & Tikpènlola & Olowondjèdja & Boerhavia sp & Ny ctaginaceae & Thérophyte & Herbe & Champs, maisons \\
\hline 7 & Elegou & Eleigou & Ceiba pentandra & Bombacaceae & Phanérophyte & Arbre & Forêts \\
\hline 8 & Eétaé & Eétaé & Celosia laxa & Amarantaceae & Thérophyte & Herbe & Champs, maisons \\
\hline 9 & Eétaé & Eétaé & Celosia trigyna & Amarantaceae & Thérophyte & Herbe & Champs \\
\hline 10 & Efooko & Efooko & Cleome viscosa & Cleomaceae & Thérophyte & Herbe & Champs, maisons \\
\hline 11 & Efooko & Efooko & Cleome rutidosperma & Cleomaceae & Thérophyte & Herbe & Champs, maisons \\
\hline 12 & Eiyo & Eiyo & Corchorus tridens & Tilliaceae & Thérophyte & Herbe & Champs, jachères, maisons \\
\hline 13 & Ebourè & Ebourè & Crassocepholum rubens & Asteraceae & Thérophyte & Herbe & Champs, maison \\
\hline 14 & Gbagodo & Gbagodo & Emilia coccinea & Asteraceae & Thérophyte & Herbe & Champs, maisons \\
\hline 15 & Gbagodo & Gbagodo & Emilia praetermissa & Asteraceae & Thérophyte & Herbe & Champs, \\
\hline 16 & Osoun & Osoun & Emilia sonchifolia & Amarantaceae & Thérophyte & Herbe & Champs, maisons \\
\hline 17 & Inonmiloofo & Inonmiloofo & Euphorbia heterophylla & Euphorbiaceae & Thérophyte & Herbe & Champs \\
\hline 18 & Djètan n'doè & Djètan n'doè & Gomphrena globosa & Amarantaceae & Thérophyte & Herbe & Champs, plantations \\
\hline 19 & Abo-adjachè & Abo-adjachè & Gynandropsis gynandra & Capparidaceae & Thérophyte & Herbe & Champs, maisons \\
\hline 21 & Eminnin odo & Eminnin odo & Ipomea aegyptiaca & Convolvulaceae & hydrophyte & Herbe & bas-fonds, mares \\
\hline 22 & Sikiti & Sikiti & Macrosphyra longistyla & Rubiaceae & Thérophyte & Herbe & Jachères \\
\hline 23 & Okpoto & Okpoto & Physalis angulata & Solanaceae & Thérophyte & Herbe & Champs, maisons, plantations \\
\hline 24 & Yèbè & Yèbè & Solanum nigrum & Solanaceae & Thérophyte & Herbe & Champs, maisons, plantations \\
\hline 25 & Ewéidou & Ewéidou & Solanum aethiopicum & Solanaceae & Thérophyte & Herbe & Champs, maisons, plantations \\
\hline 26 & Osoun odo & Imon-odo & Sparganophora spargophorus & Asteraceae & Thérophyte & Herbe & Mares, bas-fonds \\
\hline 27 & Kpakoèlèdè & Kpakoèlèdè & Stachytarfeta indica & Verbenaceae & Thérophyte & Herbe & Champs \\
\hline 28 & Ewé-èko & Ewé-èko & Sterculia tragacanta & Sterculiaceae & Phanerophyte & Arbre & forêt \\
\hline 29 & Gouré & Gouré & Talinum triangulare & Portulacaceae & Thérophyte & Herbe & Champs, plantations \\
\hline 30 & Olowo djèdja & Olowo djèdja & Trianthema portulacastrum & Aïzoaceae & Thérophyte & Herbe & Champs \\
\hline 31 & Igou & Igou & Triplochiton scleroxylon & Sterculiaceae & Phanerophyte & Arbre & forêt \\
\hline 32 & Ewouro & Ewouro & Vernonia $s p$ & Asteraceae & Thérophyte & Herbe & Champs \\
\hline 33 & Ewédudu & Ewédudu & Vernonia cinerea & Asteraceae & Thérophyte & Herbe & Champs \\
\hline 34 & Gbolo & Gbolo & Vernonia colorata & Asteraceae & Thérophyte & Herbe & Champs \\
\hline 35 & Ori & Oriri & Vitex doniana & Verbenaceae & Phanérophyte & Arbre & Champs \\
\hline
\end{tabular}




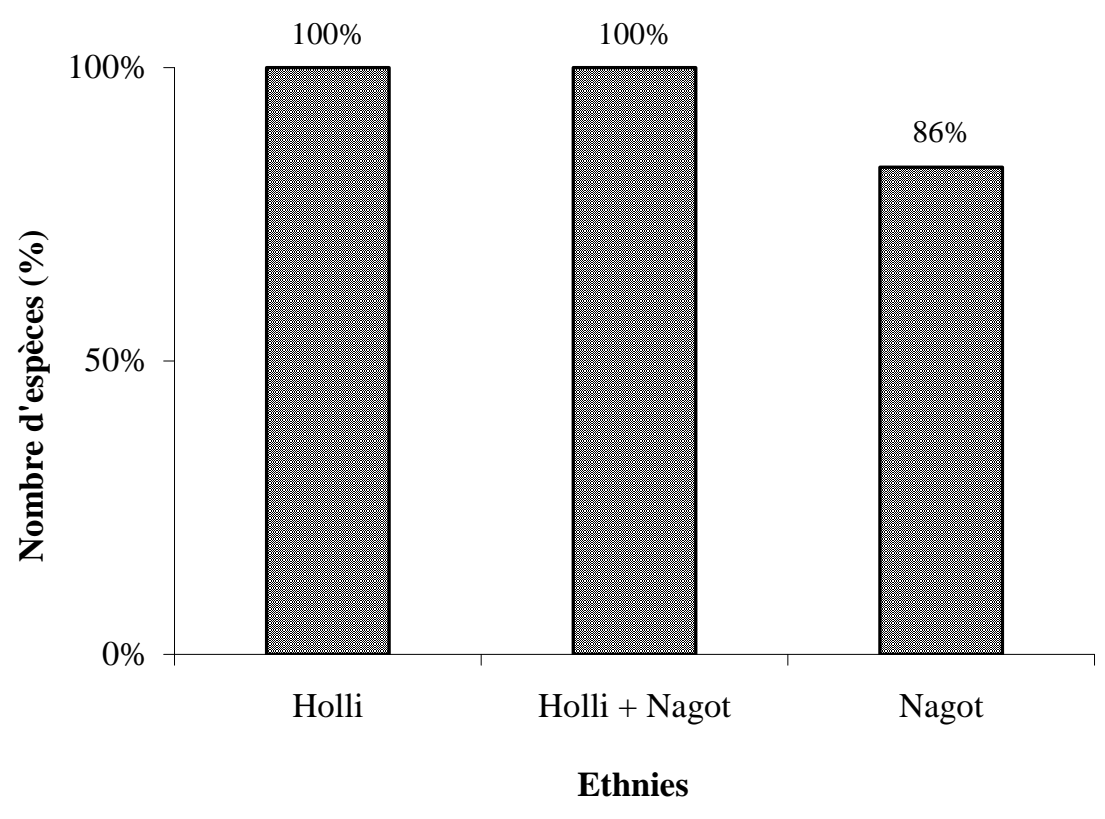

Figure 2: Spectre alimentaire par ethnie et pour les deux ethnies.

données montrent une diversité largement audessus de celle observée pour toutes les plantes alimentaires sauvages de la forêt classées de la Lama et ses zones connexes au sud-Bénin par Codjia et al. (2003). En effet, bien que située presque à la même altitude que la région de la Lama, la région de Pobè bénéficie d'un rythme pluviométrique particulier (1.180 mm de pluie/an); ce qui la singularise dans le Dahomey-gap tout en favorisant une meilleure disponibilité des ressources végétales en général. Aussi comme l'ont souligné bon nombre d'auteurs, les différentes populations n'ont pas les mêmes habitudes alimentaires; celles-ci ayant dans une large mesure une dimension culturelle et historique (Hladik et al., 1989). La région de Pobè étant culturellement plus ou moins intacte (Codjia et al., communication personnelle), les connaissances endogènes sont conservées et transmises de générations en générations sur tous les plans et même alimentaire ; ce qui est un facteur de maintien de la biodiversité des ressources biologiques quotidiennement utilisées dans divers domaines par les populations rurales.
Les thérophytes sont plus représentés (plus de $86 \%$ ) au sein de cette diversité que toutes les autres formes de vie. La région de Pobè étant caractérisée par la forte pression humaine sur les ressources forestières, ce sont les thérophytes héliophyles strictes qui sont plus diversifiés et ceci dans les milieux ouverts que sont les champs, les végétations des jardins et les jachères. Cette tendance de la plus grande diversité des espèces de légumes sauvages dans les zones dégradées est bien confirmée par Assogbadjo (2000) dans région de la Lama au sud du Bénin. Le rythme pluviométrique exceptionnel vient encore comme un facteur de leur foisonnement.

L'ensemble de ces espèces connaît une période de disponibilité assez large au cours de l'année. Même en grande saison sèche, au moins 13 espèces de légumes sont disponibles toute la saison et 18 autres partiellement pendant cette période. Cette région étant par nature une région forestière, la période de soudure qui, en zone tropicale sèche, est toujours difficile à vivre, n'existe 


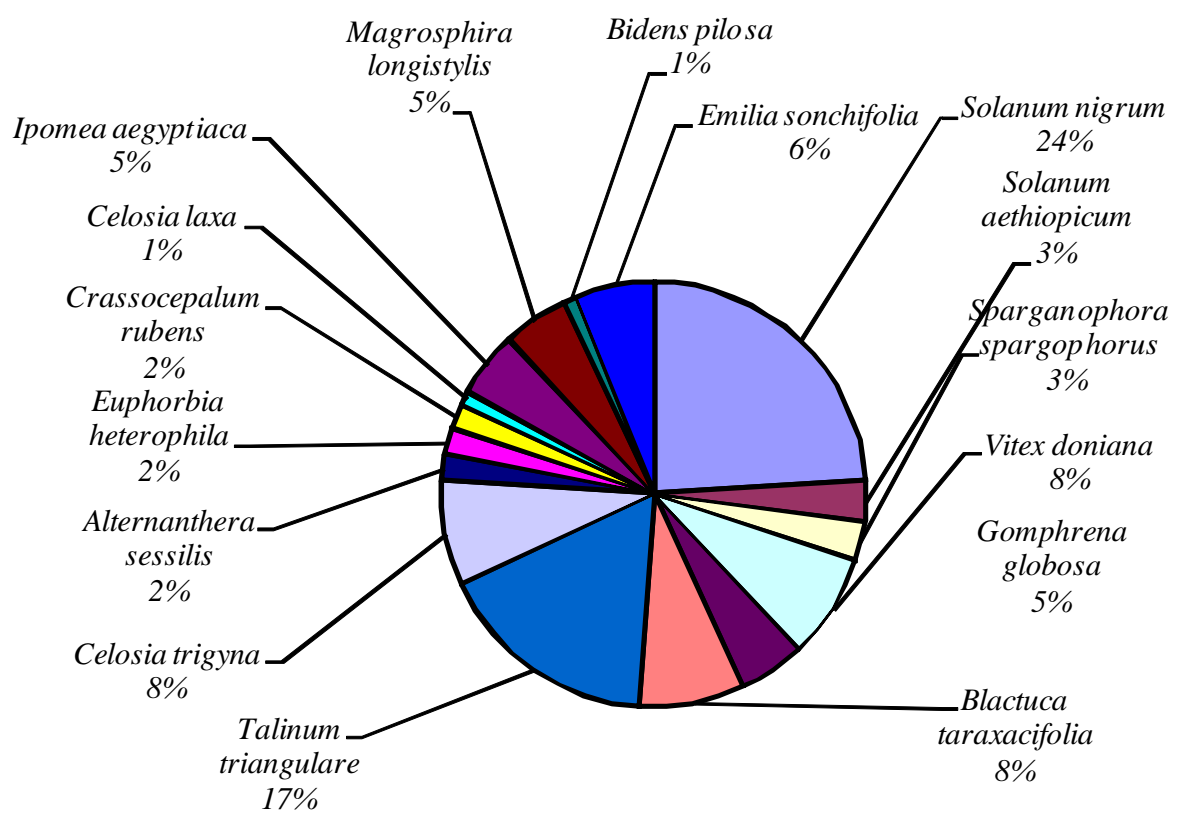

Figure 3a: Importance des espèces de légumes sauvages consommées par les populations Holli de la zone d'étude.

pratiquement pas (Hladik et al., 1989). Ces espèces ne sont pas utilisées pour couvrir les déficits de calories en périodes de soudure seulement comme l'ont mentionné Herzog (1992) et Bergeret (1986) pour les régions du sahel. Loin d'être un moyen d'adaptation aux conditions de pénurie alimentaire; elles ont parfaitement intégré les habitudes alimentaires des populations qui les utilisent quotidiennement.

Toutefois, les résultats montrent que les populations Holli ont un spectre alimentaire plus large que les Nagot de la même région. En plus de tout ce que les Nagot consomment, les Holli consomment 6 espèces (Amarantus sp. Euphorbia heterophilla, Boerhavia sp, Alternanthera sessilis, Trianthema portulacastrum et Bidens pilosa) qui sont disponibles partout dans la région de Pobè. En effet, ces espèces auraient intégré les habitudes alimentaires de ces populations lors de la sécheresse des années 80 où les terres des basses vallées seules offraient des conditions propices à ces espèces. Aujourd'hui, la consommation de ces espèces se fait sans souvenir des conditions qui l'ont engendré ; ce qui de ce fait a élargi le spectre alimentaire des Holli ainsi que la connaissance du milieu et de ses potentialités par ces derniers.

Dans de tels écosystèmes où les espèces recensées sont vivaces et presque pérennes toute l'année, on peut postuler que c'est un champ expérimental très apprécié pour les études tentant à la «domestication » (culture et étude phénologique) de ces espèces. Ensuite, une fois les connaissances scientifiques de cultures acquises et transférées en milieu paysan, cela constituerait un atout économique important pour ces populations.

\section{Conclusion}

La région de Pobè au sud-est de Bénin connaît une diversité non négligeable en ce qui concerne les espèces de légumes alimentaires locales. Malgré l'introduction et l'adoption de plusieurs espèces exotiques, les 


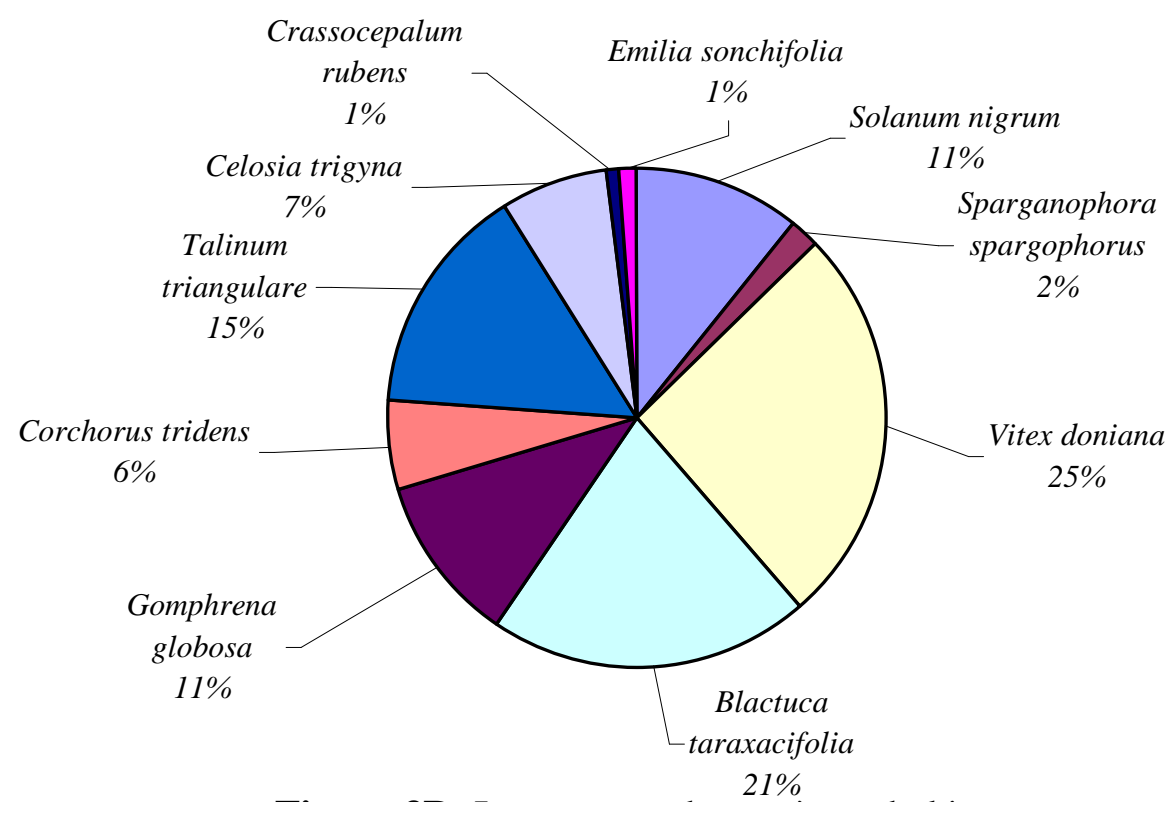

Figure 3b: Importance des espèces de légumes sauvages consommées par les populations Nagot de zone d'étude.

espèces sauvages tiennent toujours une place de choix dans les habitudes alimentaires des populations locales. Mais la dégradation sévère du couvert végétal et l'urbanisation galopante du milieu sont un facteur d'érosion des connaissances endogènes. Cette situation ne pourrait être corrigé que si l'on conduise rapidement des travaux scientifiques sur les possibilités de culture et de rentabilité économique de ces légumes cueillis jusque-là dans la nature par les populations locales. Ces étapes sont indispensables à la leur « domestication » qui est un gage pour freiner la pression sur les ressources naturelles.

\section{REFERENCES BIBLIOGRAPHIQUES}

Assogbadjo AE. 2000. Biodiversité des ressources alimentaires forestières, et leur contribution à l'alimentation des populations locales: Cas de la forêt classée de la Lama. Th. Ing. Agro.; Cotonou / FSA/UNB. Rép Bénin. P. 119.
Arbonier M. 2002. Arbres, Arbustes et Lianes des Zones Sèches d'Afrique de l'Ouest. $2^{\text {ème }}$ édition CIRAD et MNHN: Montpellier ; 573.

Bergeret A. 1986. Nourriture des cueillettes en pays sahélien. Jour. Ag. Trad. Bot. App., 33: 91-130.

Chambers R, Leach M. 1987. Trees to meet contingencies: saving and security for the rural poor. Discussion Paper 228, Institute of Development Studies; University of Sussex, Brighton.

Codjia JTC, Assogbadjo AE, Atègbo EAD. 2003. Ressources végétales de la forêt classée de la Lama et alimentation des populations riveraines. Annales des Sciences Agronomiques du Bénin, 4(2): 121-142.

Codjia JTC, Assogbadjo AE, Ekue MMR, Yorou NS. 2003. Diversité des ressources forestières alimentaires du Bénin, rôle pour les populations locales et possibilité 
de valorisation pour un développement humain durable. In Actes $d u 2^{\text {ème }}$ séminaire international sur l'aménagement intégré des forêts naturelles des zones tropicales sèches en Afrique de l'Ouest, Parakou, Bénin, 2529 juin 2001, Sokpon N, Sinsin B, EyogMatig O (eds). FSA-UAC, IPGRI, CTA, GTZ, Sida, CF; 327-370. ISBN 1659 $651 X$.

Herzog FM. 1992. Etude biochimique et nutritionnelle des plantes alimentaires sauvages dans le sud du V-Baoulé, Côte d'Ivoire. Thèse de Doctorat ès Sciences Techniques. Thèse EPFZ n 9789 , Zurich, p.123.
Hladik MC, Bahouchet S, Garine I. 1989. Se Nourrir en Forêt Equatoriale. UNESCO ; 19-25.

Sokpon N. 1995. Recherches écologiques sur la forêt dense semi-décidue de Pobè au sud-est du Bénin : groupements végétaux, structure, régénération naturelle et chute de litière. Thèse de doctorat. Université Libre de Bruxelles. p.350.

Sokpon N, Lejoly J. 1996. Les plantes alimentaires d'une forêt dense caducifoliée : Pobè au sud-est du Bénin. In L'alimentation en forêt tropicale : Interactions bioculturelles et perspectives de développement, Hladik MC et al. (eds). UNESCO; 315-324. 